\title{
FACEBOOK Y TWITTER: UN RECORRIDO POR LAS PRINCIPALES LÍNEAS DE INVESTIGACIÓN
}

\section{FACEBOOK AND TWITTER: AN OVERVIEW OF MAIN RESEARCH ASPECTS}

\author{
Laura Montero Corrales ${ }^{1}$
}

\begin{abstract}
Fecha de recepción: 23 de octubre de 2017 - Fecha de aceptación: 10 de enero de 2018
\end{abstract}
\begin{abstract}
Resumen
Este artículo analiza los antecedentes y principales aportes de la investigación en redes sociales en Internet desde el campo de la Publicidad, específicamente Facebook y Twitter. Además, se detallan estudios que hablan del fenómeno desde la producción y la recepción de los mensajes, aunado a la influencia personal en medios sociales. Se pretende que el lector reflexione sobre las diferentes disciplinas que han centrado sus esfuerzos en el estudio de la prevalencia e importancia de las redes sociales en la vida moderna y el impacto que significan para la producción, recepción y circulación de la publicidad. Existe la necesidad de realizar investigaciones sobre el cambio de las estrategias publicitarias, a raíz. del advenimiento de Facebook y Twitter.

Palabras clave: redes sociales, Facebook, Twitter, estrategias publicitarias, mensajes publicitarios.
\end{abstract}

\begin{abstract}
This paper analyzes the precedents and main contributions of the research over social media within the field of Advertising. Moreover, studies related to the production and reception of messages in social media, as well as personal influence in this medium, are referenced. The intention of this paper is to invite the reader to reflect upon all the different disciplines that have concentrated principally on the study of the prevalence and the importance of social media in modern life and the impact that they have on the publicity production, reception and traffic. There is a need to conduct research on the change in advertising strategies, as a result of the advent of Facebook and Twitter.
\end{abstract}

Key Words: social media, Facebook, Twitter, advertising, publicity messages.

\section{Introducción}

La noción de comunicación digital tiene un valor fundamental en el cambio del paradigma de las estrategias publicitarias que implementan las organizaciones. En los últimos años, Internet ha permitido que los ciudadanos y organizaciones interactúen a través de plataformas que posibilitan una serie de prácticas de consumo, producción y circulación.

La comunicación digital global, conseguida gracias a herramientas como Internet o Wi-fi, representa el núcleo del sistema de comunicación global. Actualmente, más de mil millones de usuarios en todo el mundo usan Internet y miles de millones se encuentran conectados gracias a éste y a las comunicaciones inalámbricas. Si a esto le añadimos el desarrollo de la Web 2.0, el despliegue de esta capacidad de comunicación llegaría a ser total. Por lo tanto, me voy a referir a esta interacción entre el proceso creativo y la Web 2.0. (Castells, 2009, p.2)

1 Escuela de Ciencias de la Comunicación Colectiva, Universidad de Costa Rica, laura.monterocorrales@ucr.ac.cr 
Partiendo de este hecho, surge la inquietud de analizar los antecedentes y los principales aportes de la investigación en redes sociales en Internet desde el campo publicitario, así como los estudios que analizan el fenómeno desde la producción y la recepción de los mensajes, aunado a la influencia personal en redes sociales.

Es por esto que el siguiente texto tiene como principal objetivo que el lector visualice de manera rápida cómo diferentes campos disciplinarios han concentrado sus esfuerzos en estudiar el advenimiento de redes sociales como Facebook y Twitter y su impacto en la producción, recepción o circulación de la publicidad, dejando de lado el estudio del avance del paradigma digital en la publicidad y su efecto en el diseño de los discursos publicitarios de las marcas en redes sociales, así como la participación de los líderes de opinión en la circulación de los mismos.

\section{Organización del artículo}

1. Panorama de investigaciones actuales sobre redes sociales y Facebook y Twitter en particular.

2. Análisis de las investigaciones en redes sociales desde la producción del mensaje, la recepción del mensaje y la influencia personal, dentro del campo publicitario académico, de las consultoras e investigadoras de mercado.

3. Mapeo general de énfasis de investigación en Facebook y Twitter.

El periodo del desarrollo del análisis se realiza del 2010 al 2015. Al ser un fenómeno que se ha traducido en diversos tipos de investigaciones, se tomaron revistas de América Latina de los últimos 5 años, tanto del campo académico y profesional. Además se incluyeron revistas de Estados Unidos, España, México, Nueva Zelanda y Reino Unido, donde se encontraron datos estadísticos importantes y planteamientos teóricos relevantes en cuanto a al tema de marcas y redes sociales.

En total se analizaron 15 fuentes:

1. Journal of Business Communication (Estados Unidos)

2. Journal of Computer-Mediated Communication (Estados Unidos)

3. Journal of Broadcasting \& Electronic Media (Estados Unidos)

4. Young Adult Library Services Journal (Estados Unidos)

5. Revista Comunicar (España)

6. El profesional de la información (España).

7. Revista Internacional de Relaciones Públicas (España)

8. Revista Latinoamerica de Comunicación Social (España)

9. Revista Comunicación y Sociedad (México)

10. Grup de Recerca 39; Interaccions Digitals (Flacso México. Barcelona / México DF.)

11. English Teaching: Practice and Critique (Nueva Zelanda).

12. The International Communication's Virtual Conference (Estados Unidos).

13. The Pew Internet; American Life Project Journal of Advertising Research (Estados Unidos).

14. Convergence: The International Journal of Research into New Media Technologies (Reino Unido).

15. Journal Of Database Marketing; Customer Strategy Management (Reino Unido).

Así como las revistas British Journal of Educational Technology (United Kingdom), Revista Latina de Comunicación Social (España) y el Journal of Interactive Advertising (Estados Unidos) donde se encontraron la mayor cantidad de artículos relacionados con el tema. 
El análisis a su vez se sustenta en los conceptos planteados desde el campo de la comunicación digital por autores como: Miquel Alsina, Jean Baudrillard, Danah Boyd, Manuel Castells, Michel de Certau, Pierre Levy, Marshall McLuhan, Serge Prouxl, Carlos Scolari y Eliseo Verón.

Las investigaciones en redes sociales se caracterizan por destinar la mayoría de sus esfuerzos a estudiar los contenidos y las prácticas de los consumidores. Han sido varias las disciplinas que han mostrado interés en las redes sociales y que las han estudiado desde diferentes ópticas, implementando diferentes metodologías. El mapeo general sobre los énfasis investigativos sobre Facebook y Twitter, a la vez que propone cinco posibles enfoques como a) redes sociales y dinámicas de aprendizaje, b) redes sociales y medios de comunicación, c) gestión de contenidos en redes sociales, d) redes sociales y recepción y consumidores, d) estrategias y redes sociales, entre otros; los cuales permitirían realizar investigaciones concretas para estudiar los proceso de comunicación en Facebook y Twitter.

\section{Los estudios sobre redes sociales en Internet para el campo de la publicidad}

Es en el campo de la educación desde donde se ha originado la mayoría de los estudios sobre el uso de las redes sociales, con el objetivo de incorporar estas plataformas de comunicación en el proceso de transmisión de conocimiento (Cobo y Pardo, 2007; Bassford e Ivins, 2010; Reid, 2011 \& Horn, 2011). Destaca el caso de Planeta Web 2.0. Inteligencia colectiva o medios fast food, en donde los investigadores Cobo y Pardo (2007) concluyen que las nuevas aplicaciones web de uso libre y de reciente masificación estimulan la generación y transferencia de conocimientos entre individuos y que el desafío está en que los docentes logren generar experiencias de aprendizaje y motiven la interacción entre estudiantes. Un ejemplo de este uso se menciona en Bassford e Ivins (2010), quienes hablan de Facebook como una herramienta que ha posibilitado este ambiente de cooperación e interacción, en Encouraging formative peer review via social networking sites. Estos autores definen esta red como un instrumento muy útil para compartir fotos y generar discusión, pero mencionan la limitante de que el estudiante está sujeto a tener una cuenta en esta red social si quiere participar de las diferentes actividades que se realizan en la plataforma. Este es el mismo caso que podría darse con un usuario que quiera interactuar con las marcas, para el cual la única opción de contacto que encuentra en Internet sea vía Facebook.

Por otra parte, Horn (2011), en Online Marketing Strategies for Reaching Today's Teens, menciona que Facebook es un excelente espacio de contacto con los estudiantes. La autora indica que las bibliotecas escolares que tienen un perfil en esta red social pueden crear y comunicar invitaciones a eventos, compartir videos sobre libros, publicar novedades sobre nuevos libros, responder sobre consultas relacionadas con horarios de atención y tener una presencia continua en la vida de sus usuarios adolescentes.

Lo anterior muestra cómo el campo de la educación ha sido pionero en integrar las redes sociales como objeto de estudio de sus investigaciones. Esto ha llevado al campo de la comunicación a generar espacios de discusión y análisis para investigaciones orientadas a entender el papel de las redes sociales en los procesos de comunicación y en el diseño de estrategias publicitarias.

En este campo, existen diversas líneas de estudio, como el uso que han hecho de Facebook y Twitter los medios de comunicación (García, 2011), los medios de comunicación españoles en Facebook (Noguera, 2010), el uso que han hecho las radioemisoras de Twitter (Greer y Ferguson, 2011) y hasta el uso por parte de las empresas puertorriqueñas de Facebook y Twitter (Gómez y Soto, 2011). Se puede observar con claridad un interés particular en identificar las empresas o medios de comunicación que han incluido en sus estrategias y en redes el uso de los medios sociales, además de una necesidad de describir los protocolos de uso por parte de ellas. 
Uno de los trabajos que nos genera más interés es el de Boyd y Ellison (2008), llamado Social Network Sites: Definition, History, and Scholarship, en el cual las autoras explican que la mayoría de los sitios sociales dan apoyo al mantenimiento de las ya existentes redes sociales y otros sitios permiten conectar a los usuarios según intereses comunes, opiniones políticas o actividades. Estos sitios también varían en la medida en que incorporan nuevas informaciones y herramientas de comunicación, tales como la conectividad móvil, la creación de blogs y la práctica de compartir fotos y videos. El uso de estas herramientas ha permitido a las redes sociales influir en la opinión pública y en los hábitos de consumo de la sociedad. En general, estas investigaciones han definido a Facebook y Twitter como posibles espacios que estimulan la generación y transferencia de información entre individuos participativos y productores de contenidos originales, dispuestos a interactuar con las marcas.

\section{Desde la producción de los mensajes}

Observamos una amplia oferta de investigaciones enfocadas en el estudio de la producción de los mensajes y pesquisas que incluyen tanto el análisis de lo que dicen los medios de comunicación en Twitter (Lin y Peña, 2011), como el contenido de varias marcas en Twitter (Kwon y Sun, 2011), los mensajes de las celebridades en Twitter (Boyd y Marwick, 2011), e incluso el estudio de los mensajes de las organizaciones en Facebook (Park, Rodgers \& Stemmle, 2011). Relacionado con este último tema, se menciona que las comunidades virtuales de marca pueden diferenciarse dependiendo de si son de Facebook o Twitter:

\footnotetext{
Los usuarios de Facebook se conectan con sus amigos (Boyd y Ellison 2007), y al convertirse en amigos con otros usuarios tienen acceso al contenido de sus amigos y pueden publicar información acerca de asuntos personales, enviar felicitaciones de cumpleaños, o comentarios sobre las actualizaciones de estado (Parr 2010). Los usuarios de Twitter en lugar de eso comparen sus observaciones de sus acciones en su entorno, información sobre un evento o sus opiniones sobre un tema determinado (Smith, 2010), y eligen la información que desean recibir (por ejemplo, noticias, enlaces) y las marcas que desean seguir (Parr 2010). (Kwon y Sun, 2011, p.4)
}

Siguiendo en esta línea, podemos destacar el estudio de Kwon y Sun (2011), llamado Follow Me! Global Marketers' Twitter Use. En este estudio, se examinó una muestra de 333 miembros de comunidades virtuales de marca en Corea. Los autores observaron que los miembros de una comunidad de marca tienen seis motivos sociales y psicológicos para pertenecer a ella, por ejemplo: utilidad personal, búsqueda de entretenimiento y búsqueda de información. Estos usuarios son capaces de producir contenidos por medio de la combinación de la radio y web, video y espacio público, televisión y celular o cualquier otra combinación multimedia, por lo que reciben el nombre de "prosumidores". Por esto, los autores recalcan que es necesario que las marcas escuchen lo que los consumidores tienen que decir, en lugar de simplemente "empujar" o compartir mensajes publicitarios, sin tener en cuenta lo que los clientes piensan, sienten y desean. Además, reconocen que, mediante la comunicación y la interacción con los consumidores, las marcas pueden transmitir sus personalidades y características.

Por otra parte, rescatamos del estudio de Lipsman, Mudd, Rich y Bruich (2012), How Brands Reach (and Influence) Fans Through Social-Media Marketing, en el que se concluye que el contenido de las marcas en las redes sociales puede adoptar muchas formas, entre ellas, contenido compartido directamente por las marcas, contenido re-compartido por los seguidores y contenido comercializado de manera social por medio de dos herramientas pagas de Facebook: los anuncios publicitarios y las historias patrocinadas.

Asimismo, el estudio Stimulating user activity on company fan pages in online social networks, de Huber, Landherr, Probst, y Reisser (2012) midió si los esfuerzos de las empresas en estimular la actividad del usuario en los fan page o páginas de fans son exitosos o no. Los autores observaron 
que las empresas pueden estimular la actividad y compromiso de los usuarios, si toman como base el número de seguidores totales de sus páginas de fans, mezclan las publicaciones que ellas realizan con elementos audiovisuales, fotográficos y lingüísticos y si, a su vez, realizan comentarios en las publicaciones que hacen.

De esta manera, podemos asegurar que la interactividad se convierte en un atributo diferenciador de estas dos redes sociales con respecto a otras acciones de comunicación en Internet, ya que es un rasgo que permite a los sitios de redes sociales facilitar la comprensión de los consumidores, aumentar el "boca a boca" entre redes interpersonales y mejorar la autogestión de comportamientos de los consumidores (Hyojung, Rodgers \& Stemmle, 2011). Los consumidores buscan información sobre un producto o servicio primero en las redes sociales -en lugar de un periódico o un sitio web-. Según Pérez-Later, Blanco y Sánchez (2011), "los consumidores están impulsando los mercados, y a su vez, Internet ha cambiado la forma en que los consumidores aprenden, obtienen información y se relacionan entre sî" (p.70), lo que provoca que los discursos publicitarios de las marcas busquen la eficacia persuasiva aplicada a las nuevas tecnologías como los medios sociales.

Sin embargo, esta no es una tarea fácil. Según lo confirman algunos estudios, existe una dificultad para lograr que los usuarios consuman anuncios en Internet. Según Chi (2011), los gerentes de marca suelen buscar dos tipos de comunicación digital: la publicidad interactiva y las comunidades virtuales de marca (p.2). La publicidad interactiva en el campo de la comunicación digital es definida por Romero y Fanjul (2010) como: "los mensajes publicitarios que están emplazados en una página web, en un mensaje de correo o cualquier soporte similar que suelen tener un carácter estático o presentar elementos animados para llamar la atención del usuario" (p. 127). Así las cosas, se puede afirmar que las marcas podrían aprovechar mejor las posibilidades de comunicación que brindan estas plataformas. Por ejemplo, pueden abrir un perfil en alguna de estas dos redes sociales, diseñar los materiales gráficos y completar la información general relacionada con sus características y naturaleza de negocio para que el perfil sea visualmente atractivo. En segundo lugar, pueden producir y compartir contenidos que les permitan generar suficiente información de interés para los usuarios que cuentan con un perfil en uno de estos dos espacios. Por último, para activar el proceso de interacción, las marcas pueden buscar, a través de diferentes estrategias, que estos consumidores se conviertan en seguidores de sus perfiles.

Para la mayoría de los autores, lograr o motivar la interacción es el objetivo principal de las redes sociales. En términos generales, la utilidad de estos datos radica en el hecho de contar con información que describa las características tecno-comunicativas de Facebook y Twitter y, a su vez, posibles formatos de los discursos en estas dos redes sociales por parte de las marcas.

Sin embargo, queda clara la necesidad de incluir el papel del receptor en la construcción del objeto de estudio para poder analizar la interacción, en este caso entre marcas y personas. En este contexto, algunas investigaciones que han surgido desde la desde óptica de la recepción, han brindado una serie de datos valiosos para el campo, pero han obviado el estudio de la interacción del usuario con la empresa, organización o la marca.

\section{Desde la recepción de los mensajes}

Actualmente, la crisis de los targets o el público meta y el surgimiento de un nuevo lenguaje que entremezcla los discursos publicitarios y periodísticos en redes sociales, confirma el hecho de que los consumidores son heterogéneos y productores, a la vez que los discursos se presentan de manera multimodal. Para Canclini (2011), la noción de "prosumidor" apareció en la última década, a partir de que en muchos procesos culturales y comunicacionales ya no se veía el circuito secuenciado de producción, circulación y consumo, sino que había cierta circularidad descentrada en la que los receptores 
del mensaje, eran al mismo tiempo son consumidores y podían modificarlo, reintroducirlo en las redes sociales o ponerlo en otro lugar (cit. Scarpelli, 2011).

En este marco, existen diferentes estudios, autores y especialistas en el tema que establecen que ambas redes están ayudando a los usuarios a tomar un rol más importante, una posición en la que su capacidad de información, decisión e influencia sobre las marcas y otros consumidores es mucho más relevante.

Las estadísticas muestran un crecimiento continuo en la cantidad de usuarios que cuentan con un perfil en Facebook. Según el sitio web insidefacebook.com, durante octubre de 2014, el 40 por ciento de los usuarios de esta red social estaban entre las edades de trece y veinticinco años, lo que equivale a aproximadamente 728 millones personas en el mundo. Si bien es cierto, los consumidores tienen la capacidad de convertirse en productores de contenido a favor o en contra de una marca (es decisión de ellos a qué marcas siguen en redes sociales), pero esto no evita que el consumidor también esté sujeto a la constante publicación de información que se entremezcla con las publicaciones de los amigos de los usuarios; ya que la dinámica de Facebook y Twitter permite que los consumidores lean los mensajes de un amigo y de una marca al mismo tiempo. Esto ha llevado a las marcas a utilizar lenguajes y formatos tan "naturales" como los de las personas. Por esta razón, es normal que en algunos casos las fronteras entre los discursos publicitarios y periodísticos se difuminen y sea más difícil para las marcas desarrollar estrategias de comunicación bajo los esquemas tradicionales de los formatos publicitarios ${ }^{2}$. Una hipótesis que podemos plantear es que las marcas han generado un nuevo modelo de relación o interacción con los usuarios, respetando sus intereses económicos a la vez que adoptan nuevas formas de comunicarse que podrían confundir al consumidor.

Los estudios desde la recepción han adoptado diferentes metodologías, problemáticas y categorías. Se rescatan las investigaciones sobre los nativos e inmigrantes digitales (Piscitelli, 2006), contenido y audiencias (Ognyanova, 2011), relación de las comunidades con las audiencias (Ang, 2011) y la recepción de los estudiantes universitarios (Monge \& Olabarri, 2011). También existen diferentes investigaciones a nivel comercial y profesional que han buscado determinar los hábitos de consumo de las personas en redes sociales.

Al ser los medios sociales un espacio donde los usuarios son alentados a conectarse, conversar, crear y colaborar (Ang, 2010), este receptor ha tenido que utilizar redes sociales como Facebook o Twitter para facilitar la interacción social con amigos, familia, compañeros de trabajo, conocidos, desconocidos, instituciones del estado, empresas, o simplemente para comunicar lo que piensa o siente. Un mayor índice de relación entre las personas, acceso a herramientas que les permitan la creación de comunidades y la posibilidad de los usuarios de apropiarse de los contenidos publicitarios por fuera de los circuitos tradicionales, son algunas prácticas de las comunidades en redes sociales. Y es a partir de estos medios de comunicación en los que el sujeto despliega su identificación con tal o cual candidato político, equipo de fútbol al que pertenece o producto que consume (Van Peborgh, 2010). Esto constituye una diferencia con respecto a los medios escritos, en donde los receptores no tienen la posibilidad de interactuar "al instante", directamente con el periodista que escribió la nota. Ante este planteamiento, Pérez-Later, Blanco y Sánchez (2011) destacan que:

Los consumidores están impulsando los mercados, y el Internet cambia la forma en que los consumidores aprenden, obtienen información y se relacionan entre sí. La publicidad trata de dar rienda suelta a la participación del consumidor en todos los puntos de contacto de las marcas. Las redes sociales se han convertido en una herramienta de investigación importante y una forma de comunicarse directamente con los consumidores. Los planificadores

2 Autores como Noguera (2010), establecen que: "A raíz de la socialización de los contenidos, los contactos se pueden denominar "amigos", "fans" o "seguidores" (p.179). Para fines de la investigación, se utilizara el término seguidores para ambas redes, ya que en Twitter no se obtienen "amigos" ni "fans" sino followers (Faina, 2012, p.66). 
estratégicos en la publicidad las utilizan para conocer al consumidor, tratando de profundizar en el conocimiento de los usuarios y ésta es un área de investigación. En las redes sociales, aunque su crecimiento de audiencia ha sido espectacular, todavía hay importantes dudas del modelo de negocio. (p. 70)

Algunas preguntas que pueden derivar de la observación anterior son: ¿Cuáles son los nuevos modelos de recepción, ¿cuál es su impacto?, ¿cómo es este usuario digital? Para intentar responder a estas preguntas es necesario especificar ciertas ideas relacionadas con lo que hacen las audiencias con estos mensajes y cómo estos influyen en su vida, así como la relación del consumidor con estas marcas en redes sociales y la cotidianidad en el espacio digital.

Boyd (2014) menciona, en su libro It's Complicated. The Social lives of networked teens, que los jóvenes nacidos entre 1980 y 1990 son "nativos digitales" y los nacidos antes de 1979, "inmigrantes digitales", lo cual ha generado connotaciones tanto positivas como negativas. La noción de "nativo digital" ha sido rechazada por la mayoría de los investigadores, pero el público en general sigue abrazando el término. A raíz de esto, la autora sugiere la siguiente descripción sobre nativos digitales, con el fin de brindar una acepción mucho más precisa y menos polémica:

Los nativos digitales comparten una cultura global común que se define no por la edad, en el sentido estricto, sino por ciertos atributos y experiencias relacionadas con la forma en que interactúan con las tecnologías de la información, la información en sí, unos a otros, y otras personas e instituciones. Los que no han nacido digital, pueden ser tan conectados, si no más, que sus contrapartes más jóvenes. Y no todo el mundo que nace desde, digamos, 1982, pasa a ser un nativo digital. (2014, p. 196)

Concordamos con el planteamiento de Boyd, por lo que utilizamos el término "jóvenes" en redes sociales y asumimos que estos jóvenes realizan un sinfín de prácticas en Internet y en medios sociales, unos con más intensidad y constancia que otros. De manera tal que nuestro interés, más que profundizar en la noción de nativos digitales, jóvenes o las prácticas en Internet de estos usuarios, es identificar situaciones en Facebook y Twitter relacionadas con las marcas, sus perfiles y sus estrategias y el uso que estos jóvenes les dan a los contenidos, así como su opinión en relación con el tema de líderes de opinión digital.

Vemos necesario apoyarnos en los resultados de distintos estudios sobre marcas y jóvenes para tener un marco de referencia; aprovechando el incremento en la participación de las consultoras e investigadoras de mercado, producido gracias a una mayor elucidación en el análisis de las prácticas de las personas en redes sociales.

Diferentes firmas y consultoras en investigación de mercados y opinión pública, como Pew Research Center (Estados Unidos), UNIMER Centroamérica, The Cocktail Analysis (España y México), y comScore (Estados Unidos, Europa, Latinoamérica y Asia), se han dado a la tarea de incluir el estudio de las redes sociales en su agenda de pesquisas; con lo que han definido cuántas personas utilizan Facebook y Twitter, cuántas de esas personas son hombres o mujeres, cuántos años tienen, cuáles otras redes sociales utilizan y cuáles son las redes sociales que más usan.

Por ejemplo, comScore, en su último informe acerca de redes sociales en América Latina (junio, 2012), estableció que 114,5 millones de personas en Latinoamérica visitaron Facebook y 27,4 millones ingresaron a Twitter; lo que los llevó a concluir que Latinoamérica es la región más involucrada en redes sociales a nivel global. Por otro lado, Pew Research Center, en su estudio "The tone of life on social networking sites" (Lenhart, Smith y Rainie, 2012), comprobó que el clima social y emocional de las redes sociales es muy positivo y se considera como un lugar donde los adultos reciben satisfacciones personales mucho más altas que en otros espacios. Por otra parte, otro estudio de la misma consultora, que trabajó específicamente el uso de Twitter (2012), estableció que, de una muestra de 4507 estadounidenses, el 15\% de los encuestados utilizaron Twitter todos los días durante el 2011.

Como podemos ver, los estudios de comScore o Pew Research Center describen un aumento en el nivel de avance de las redes sociales en las prácticas cotidianas de socialización y consumo de 
información. Es importante recordar que estos son estudios con fines económicos que las consultoras llevan a cabo y comercializan entre las empresas y el mercado y que, en la mayoría de los casos, no profundizan en gran medida sobre otros aspectos de la comunicación e interacción entre usuarios y otros actores, como las marcas.

Por lo tanto, al no contar con este tipo de análisis, se rescatan otras investigaciones que, si bien no hablan directamente sobre esta relación entre marcas/consumidores en Facebook y Twitter, brindan un aporte interesante en torno a la gestión de los contenidos en estas dos plataformas de comunicación. En general, estos estudios describen las diferencias entre Facebook y Twitter, realizan pequeñas aproximaciones sobre los tipos de discursos que existen en cada una de estas redes sociales y muestran, con ejemplos, recomendaciones dirigidas a las marcas para que administren de mejor manera los contenidos que publican.

El primer estudio, La $4^{a}$ Oleada Observatorio de Redes Sociales en España (2012), realizado por The Cocktail Analysis, llevó a cabo un total de 1.304 encuestas a usuarios entre 16 y 45 años y 26 entrevistas en profundidad. Algunos resultados con respecto a Facebook fueron: la confirmación de esta red social como espacio en donde el papel de las marcas es más relevante (un 65\% de los encuestados afirma haber tenido contacto con alguna empresa o marca a través de esta red) y la identificación de una práctica de uso de esta red social por parte de los usuarios (el $62 \%$ indicó que el objetivo por el cual se relacionaban con las marcas era para obtener descuentos y promociones). Podemos afirmar que este último dato es habitual en el comportamiento de los usuarios en relación con la publicidad de las marcas pero que ha cambiado en redes sociales, ya que no todas las marcas en estos espacios producen discursos promocionales, pues la mayoría adopta un tono de comunicación más social, amigable e informativo.

Con respecto a Twitter, se rescatan dos hallazgos importantes: el 32\% de los encuestados indicó que tuvo contacto con una marca en esta plataforma y el $61 \%$ expresó que se relacionaba con esa marca porque le parecía interesante. ¿Qué significa que una marca sea interesante?, ¿por qué siguen a las marcas en esta red social? Sobre este punto el estudio indicó que:

La presencia de las marcas resulta natural para los consumidores, debido al hecho de ser un medio gratuito (que debe financiarse) y a que son un tema de conversación habitual para ellos mismos (las novedades, los productos que gustan, los anuncios, etc.). Sin embargo, para establecer una relación con ellas, se demanda la obtención de beneficios tangibles y evitar una serie de elementos que causan rechazo: resultar intrusivo respecto a los datos personales, la presencia excesiva de publicidad o actualizaciones, y un tono de comunicación poco adecuado (ya sea por ser demasiado informal, poco creíble, o demasiado institucional, muy lejano). (The Cocktail Analysis, 2012, p.2)

Aunado a esto, también nos resulta importante conocer la opinión de los costarricenses acerca de la naturalidad de la presencia de las marcas en las redes sociales que utilizan, qué piensan sobre la presencia excesiva de publicidad y si consideran que las marcas utilizan un lenguaje con un tono poco creíble.

El primer estudio, de la consultora digital Findasense (2015), realizado a 100 jóvenes costarricenses entre 14-19 años, con el objetivo de explorar el comportamiento de la juventud en el entorno digital, identificó que los jóvenes ticos utilizan Twitter para seguir perfiles de usuarios famosos, como un diario personal, para ver lo que pone la gente, consumir noticias o expresarse. Según los encuestados, Facebook sigue siendo como "la fiesta en la que hay que estar para no perderse nada".

Otro hallazgo del estudio tiene relación con los líderes de opinión. Ante la pregunta "¿Qué piensas de los influencers cuando te recomiendan algo relacionado con una marca?" los jóvenes ticos entrevistados indicaron que no les gusta cuando hablan de marcas o sienten que seguramente lo están haciendo como parte de su trabajo, no algo espontáneo.

Por último, el estudio plantea una ficha del Social Teen en Costa Rica (figura 1), que nos permite conocer un poco más las características de este usuario, algunas preferencias a nivel de consumo digital, uso de tecnologías y motivaciones: 
Figura 1

El Teen en Costa Rica

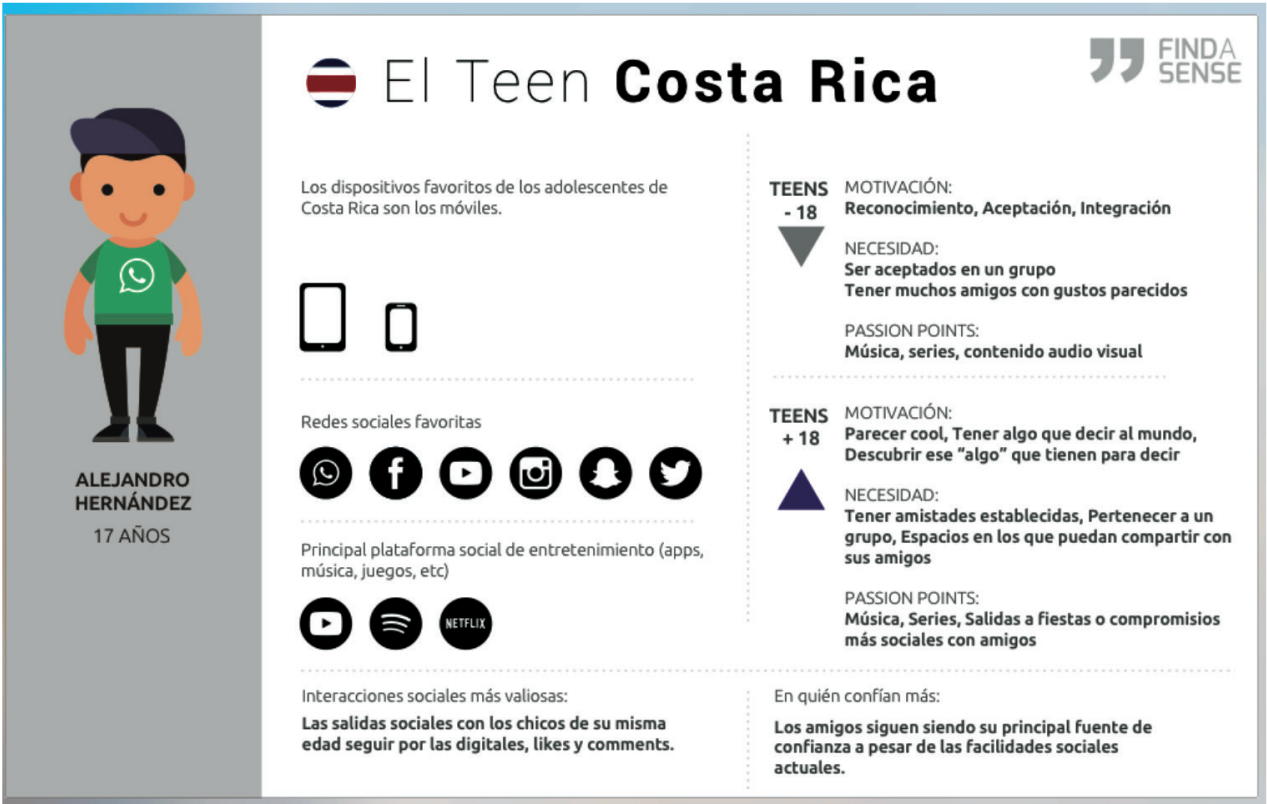

Fuente: estudio FINDOUT de Findasense.cr (octubre de 2015).

La imagen anterior nos brinda datos que podrían ser de gran utilidad para las estrategias publicitarias que las marcas desarrollan en redes sociales, a la vez que vislumbra la necesidad de profundizar en el estudio etnográfico de los usuarios en redes sociales o los nativos digitales.

Por otro lado, incluimos un segundo estudio realizado a una muestra de 4753 estudiantes de la Universidad del País Vasco, que analiza la relación que los universitarios mantienen con las marcas en Facebook. Algunos hallazgos que podemos rescatar son:

1. A los universitarios, la presencia de las marcas no les produce rechazo. El 64,8\% de ellos están de acuerdo con que las marcas estén en Facebook.

2. El 38,3\% de los entrevistados ha revisado el contenido que se publica en la página de una marca.

3. Las acciones que exigen un mayor esfuerzo son las que obtienen menor apoyo. El $15,2 \%$ ha publicado un comentario y el $11 \%$ ha publicado información por iniciativa propia.

4. Las razones de más peso para hacerse fan de una marca son: los descuentos como motivo de relación (96\%), recibir regalos o participar en concursos con premios (93\%) y recibir información de nuevos productos $(90 \%)$.

En Costa Rica no contamos con estudios que nos muestren hallazgos similares a los planteados por el estudio anterior; sin embargo, esperamos que la encuesta realizada nos permita conocer un poco más sobre este tema.

\section{Influencia personal en redes sociales}

Desde una perspectiva convergente, estos procesos de comunicación digital abarcan una serie de parámetros para su comprensión que clasifican en dimensión empresarial, tecnológica, profesional y 
comunicativa (Salaverría, 2003). Estos procesos de convergencia han incorporado nuevos perfiles profesionales y, al mismo tiempo, han rediseñado las funciones de los roles tradicionales (Scolari, 2008); como el rol del líder de opinión, el cual consideramos que logra cierto estatus de notoriedad a nivel digital, pero no es tomado en cuentan dentro del proceso de producción de la comunicación entre marcas y usuarios en redes sociales.

Debido a que la investigación de la influencia personal en redes sociales en Costa Rica es incipiente, aún no se cuentan con pesquisas que trabajen este tema o desarrollen como línea de investigación principal el análisis de la influencia digital. Sin embargo, podemos recatar estudios internacionales, como la investigación Líderes de opinión en Colombia, Venezuela e Irán. El caso de los 20 usuarios más vistos en Twitter (Said y Arcila, 2010). De acuerdo con este análisis, las redes sociales están contribuyendo a la formación de nuevos líderes de opinión. Los autores centraron su análisis en Twitter y la lista de los 20 perfiles con más seguidores en Colombia, Venezuela e Irán con el objetivo de comprender los rasgos que caracterizan los mensajes publicados en los perfiles de estos usuarios y el tipo de influencia ejercida por ellos en otros miembros de dicha red social. La investigación anterior pone en evidencia la necesidad de desarrollar investigaciones sobre líderes de opinión y procesos de comunicación en Costa Rica.

Otro trabajo a rescatar es el que realizó Sismondo (2013) sobre líderes de opinión en la industria farmacéutica. En Key Opinion Leaders and the Corruption of Medical Knowledge: What the Sunshine Act Will and Won't Cast Light On el autor propone una mirada más crítica sobre los líderes de opinión, al señalar que hay médicos que actúan como líderes de opinión clave y que se caracterizan por tener una gran población de clientes, por lo que pueden prescribir una gran cantidad de recetas al mes. Una combinación de portavoz, popularidad y simpatía.

El autor indica que tendría sentido prohibir las charlas promocionales por parte de los médicos y que esas conversaciones sean proporcionadas por los representantes de ventas, cuya labor profesional es promocionar medicamentos. Sismondo (2013) también pone en evidencia la necesidad de que estas compañías tengan una interacción más estrecha con los médicos. Se rescata el hecho de que esta industria ha hecho referencia a este aspecto y ha pulido mecanismos como las guías de uso de producto, las charlas pagadas e incluso la oferta de beneficios económicos para los líderes de opinión con el objetivo real de sacar ventaja de este flujo de comunicación y tener una retribución económica.

En general, la mayoría de los estudios son de carácter descriptivo, empírico y cuantitativo, dejando de lado el análisis integral de los tres actores: marcas, discursos y redes interpersonales. Es posible concluir que una investigación que analice cómo las marcas interactúan con los usuarios en estas dos plataformas puede mejorar la comprensión de la variabilidad de estos espacios como medios de comunicación y proporcionar ideas sobre cómo es el proceso de producción y circulación de los mensajes de las marcas y la finalidad social que los usuarios hacen de la información que se publica en estas redes.

\section{Aportes finales}

Basados en lo que hemos mencionado, proponemos el siguiente esquema como marco específico dentro del cual se pueda incorporar un estudio de las estrategias publicitarias de las marcas en redes sociales que abarque desde el consumo, la producción y la circulación, hasta el dominio teórico y el análisis comparativo entre el campo tradicional y el ámbito tecnológico.

En el área de las investigaciones sobre redes sociales, sin ánimos de querer modificar este dinámico campo en un pequeño reflejo de su realidad, podría decirse que parte de la discusión académica se produce a partir de los grandes enfoques generales que planteamos según énfasis:

1. Desde el campo de la educación, encontramos el uso de las redes sociales por parte de los docentes, implementación de las redes sociales como herramientas pedagógicas en las clases y uso de las redes sociales como herramientas de investigación. 
2. Identificación de medios de comunicación en Facebook y Twitter así como el uso de Facebook y Twitter por parte de las televisoras, periódicos y radioemisoras. Esto desde el campo de la Comunicación.

3. Desde el campo de la Informática, comunicación, publicidad, semiótica, se estudia el análisis de los mensajes de los medios de comunicación, celebridades, organizaciones y marcas comerciales en Facebook y Twitter y desarrollo de estrategias para la administración de contenidos.

4. Sobre el perfil del receptor en redes sociales (prosumidores, productores, nativos digitales). Uso de las redes sociales por parte de los universitarios y los jóvenes, prácticas de socialización entre las personas en redes sociales y prácticas de interacción de las personas en los perfiles de las empresas en Facebook y Twitter, donde participan las investigaciones del campo de la comunicación, sociología y psicología.

5. Hábitos de consumo de las redes sociales por parte de las personas. Identificación de poblaciones en redes sociales. Uso de Facebook y Twitter por parte de las empresas. Características de los perfiles de las marcas y medios de comunicación en Facebook y Twitter. Son enfoques que se estudian desde las disciplinas de los negocios, mercadeo, investigación de mercados y psicología del consumidor.

El mapeo general sobre los énfasis investigativos sobre Facebook y Twitter, propone cinco posibles enfoques como redes sociales y dinámicas de aprendizaje, redes sociales y medios de comunicación, gestión de contenidos en redes sociales, redes sociales y audiencia y mercados y redes sociales, que permitirían definir investigaciones concretas para estudiar los proceso de comunicación en Facebook y Twitter. Este trabajo muestra la necesidad de realizar investigaciones en el cambio de las estrategias publicitarias raíz del advenimiento de las redes sociales, determinar los procesos de interacción entre audiencias digitales y marcas en estos soportes de comunicación, como lo son Facebook y Twitter. Se estaría hablando de un sexto enfoque relacionado con las redes sociales, audiencias y marcas.

Por otra parte, este análisis también muestra la necesidad de realizar investigaciones sobre el cambio de las estrategias publicitarias, a raíz del advenimiento de las redes sociales, y determinar los procesos de interacción entre jóvenes y marcas en estos medios de comunicación. Podríamos hablar, entonces, de un sexto enfoque relacionado con marcas y editorialización de contenidos en redes sociales. Resulta innegable que Facebook y Twitter continúan siendo hasta la fecha las redes sociales con mayor cantidad de usuarios y las que generan un mayor interés para investigar.

Es de suma importancia conocer cuántas personas están en Facebook y Twitter y qué hacen, porque estos datos son más afines al tipo de información que necesitan los profesionales en ventas y mercadeo. Por otro lado, si bien los académicos han estudiado ciertas prácticas y usos de las personas con perfiles en Facebook y Twitter, pocos han incorporado en sus pesquisas el análisis de la interacción entre marcas y personas, de manera tal que surge la necesidad de incorporar estas dos interrogantes en el campo de la investigación en comunicación digital: ¿Cuáles investigaciones concretas podrían resultar cuando se estudia la comunicación en Facebook y Twitter, tanto desde la producción como desde recepción de los productos comunicativos? y ¿Cuál es el rol que juegan los líderes de opinión en estos procesos de comunicación digital?

\section{Referencias}

Acosta, Yanet (2010). Twitter y la comunicación gastronómica: II Congreso Internacional Latina de Comunicación Social. España: Universidad La Laguna.

Aguado, María. (2012). ¿Para qué sirven las redes sociales? Revista de Comunicación, 45, 7-9.

Alsina, Miquel. (1995). Los modelos de la comunicación. Madrid: Técnos.

Andrés, Gonzalo. (2012). Prácticas culturales en red: reflexión sobre la participación y producción de los internautas. Poliantea, 14(8). 
Auletta, Nuncia. (2009). Las empresas criollas y el mercadeo en la red. Debates IESA, 14(2), 14-20.

Barlund, Dean. (1970). A transactional systems model of communication: Implications for transactional analysis. Journal of Business Communication, 15, 65-73.

Bassford, Marie e Ivins, Jonathan. (2010). Encouraging formative peer review via social networking sites. British Journal of Educational Technology, 41(5), E67-E69.

Baudrillard, Jean. (1975). El sistema de los objetos. México: Siglo XXI.

Baudrillard, Jean. (1978). Cultura y simulacro. Barcelona: Editorial Kairos.

Baudrillard, Jean. (1998). The consumer society. London: SAGE Publications.

Belch, George y Belch, Michael. (2004). Publicidad y promoción. Perspectivas de la comunicación de marketing integral (6 $6^{\mathrm{a}}$ ed.). México: McGraw-Hill.

Boyd, Danah. (2007). Socializing digitally. Vodafone Receiver Magazine, 18, 1-6.

Boyd, Danah. (2008). Social setwork Sites: Definition, history, and scholarship. Journal of ComputerMediated Communication, 13(1), 210-230.

Boyd, Danah. (2011). To see and be seen: Celebrity practice on Twitter. Convergence: The International Journal of Research into New Media Technologies, 17, 139.

Boyd, Danah. (2014). It's complicated: The social lives of networked teens. New Haven, CT: Yale University Press.

Burgess, Jean. (2006). Hearing ordinary voices. Continuum: Journal of Media \& Culture, 20(2), 201-214.

Castells, Manuel. (2009). Creatividad, innovación y cultura digital. Revista Telos. Recuperado de https:// telos.fundaciontelefonica.com/telos/articulocuaderno.asp@idarticulo=2\&rev=77.htm

Certau, Manuel. (1980). L’invention du quotidien (Vol. 1). Paris: UGE.

Cobo, Cristóbal y Pardo, Hugo. (2007). Planeta web 2.0. Inteligencia colectiva o medios fast food. Barcelona / México DF: Universitat de Vic. Flacso México.

Del Pino, Cristina. (2011). Redes sociales, comunicación publicitaria y usuario digital en la nueva era. Revista Comunicar. 9(1), 163-174.

Facebook. (2011). Facebook info. Recuperado de_http://www.facebook.com/face book?sk=info

Faina, Joseph. (2012). Twitter and the new publicity. A Review of General Semantics, 69(1), 55-71.

Flores, Jesús. (2009). Nuevos modelos de comunicación, perfiles y tendencias en las redes sociales. Revista Científica de Comunicación y Educación, 33, 73-81.

García-De-Torres, Elvira, Yezers'ka, Lyudmyla, Rost, Alejandro, Calderin, Mabel, Edo, Concha, Rojano, Miladys, Said, Elías y Correidora, Loreto. (2011). Uso de Twitter y Facebook por los medios iberoamericanos. El Profesional de la Información, 20(6), 611-620.

Gómez, Margarita. y Soto, Ivette. (2011). Los medios sociales como una herramienta estratégica para la comunicación corporativa. Revista Internacional de Relaciones Públicas. 1(2), 157-174.

Gómez Borrero, Pilar. (2010). Prensa e internet. ¿Dónde está el negocio? Madrid: MediosOn.

Greer, Clark y Ferguson, Douglas. (2011). Using Twitter for promotion and branding: A content analysis of local television Twitter sites. Journal of Broadcasting \& Electronic Media, 55(2), 198-214.

Hoffman, Donna y Fodor, Marek. (2010). Can you measure the ROI of your social media marketing? Sloan Management Review, 52(1). Recuperado de https://ssrn.com/abstract=1697257

Horn, Laura. (2011). Online marketing strategies for reaching today's teens. Young Adult Library Services, 9(2), 24-27.

Hyojung, Park, Rodgers, Shelly y Stemmle, Jon.(s.f.) Health organizations' use of Facebook for health advertising and promotion. Journal of Interactive Advertising. 12(1), 62-77.

Hsu-Hsien, Chi. (2011). Interactive digital advertising vs. virtual brand. Community: Exploratory study of user motivation and social media marketing responses in Taiwan. Journal of Interactive Advertising, 12(1), 44-61.

Huber, Johannes, Landherr, Andrea, Probst, Florian y Reisser, Christian. (2012). Stimulating user activity on company fan pages in online social networks: ECIS 2012.

Katz, Elihu y Lazarsfeld, Paul. (1955). Personal influence. New Brunswick, NJ: Transaction Publishers. 
Klein, Naomi. (2007). No logo: El poder de las marcas. Barcelona: Paidós.

Kwon, Eun y Sun, Youngjun. (2011). Follow me! Global marketers' Twitter use. Journal of Interactive Advertising, 12,1-10.

Lipsman, Andrew, Mudd, Graham, Rich, Mike y Bruich, Sean. (2012). The power of "like": How brands reach (and influence) fans through social-media marketing. Journal of Advertising Research, 52(1), 40-52.

Lehhart, Amanda, Smith, Aaron y Rainie, Lee. (2012). The tone of life on social networking sites 2012. Pew Research Center's Internet \& American Life Project. Recuperado de http://pewresearch.org/ pubs/2189/social-networking-sites-behavior

Levy, Pierre. (2007). Cibercultura. Barcelona: Anthropos.

López, Guadalupe y Ciuffoli, Clara. (2012). Facebook es el mensjae. Oralidad, escritura y después. Buenos Aires: La Crujía.

Maestri, Mariana. (2011). Las audiencias digitales: convergencias y prácticas. En Valdettaro, S. (ed.), Interfaces y pantallas: análisis de dispositivos de comunicación. Rosario: UNR.

McLuhan, Marshall. (1996). The medium is the message. Berkeley, CA: Gingko Press.

Monge, Sergio y Olabarri, María. (2011). Los alumnos de la UPV/EHU frente a Tuenti y Facebook: Usos y percepciones. Revista Latina de Comunicación Social, 66, 1-25.

Noguera, Jose. (2010). Redes sociales como paradigma periodístico. Medios españoles en Facebook. Revista Latina de Comunicación Social, 65, 176-186.

Ognyanova, Katherine. (2011). Actors and links in the media system: Applying a network perspective to the study of media production, content and audience: The International Communication's Virtual Conference.

O'Reilly, Tim. (2005). What is web 2.0. Recuperado de http://oreilly.com/web2/archive/what-is-web-20. html

Pérez-Later, Francisco, Blanco, Idoia y Sánchez, Cristina. (2011). Social networks, media and audiences: A literature review. Comunicación y Sociedad, 24(1), 63-74.

Prouxl, Serge. (2001). Les formes d'appropriation d'une culture numérique comme enjeu d'une société du savoir: COREVI. Québec.

Romero, María y Fanjul, Carlos. (2010). La publicidad en la era digital: El microsite como factor estratégico de las campañas publicitarias on-line. Revista Científica de Comunicación y Educación, 34, 125-135.

Salaverría, Ramón. (2003). Convergencia de medios. Revista Latinoamericana de Comunicación, 81, 65-81.

Said, Elías y Arcila, Carlos. (2011). Líderes de opinión en Colombia, Venezuela e Irán. El caso de los 20 usuarios más vistos en Twitter. Comunicación y Sociedad, 24(1), 75-100.

Sánchez, Luis. (1997). Semiótica de la publicidad. Barcelona: Editorial Síntesis.

Santesmases Mestre, Miguel. (1996). Términos de marketing. Diccionario-base de datos. Madrid: Pirámide.

Scarpelli, Agustín. (2011). Google es más poderoso que las cadenas de tv o las discográficas. Revista $\tilde{N}$. Recuperado de http://www.revistaenie.clarin.com/ideas/tecnologia comunicacion/ Entrevista_Nestor_Garcia_Canclini_0_559144321.html

Scheinsohn, Daniel. (2014). La tercera gran revolución de la comunicación en las organizaciones y la inminencia del DircoN. RiHumSo - Revista de Investigación del Departamento de Humanidades y Ciencias Sociales, 2(5), 3-22.

Scolari, Carlos. (2004). Hacer clic. Hacia una sociosemiótica de las interacciones digitales. Barcelona: Gedisa.

Scolari, Carlos. (2008). Hipermediaciones. Elementos para una teoría de la comunicación digital interactiva. Barcelona: Gedisa. 
Strand, John. (2011). Facebook: Trademarks, fan pages and community pages. Intellectual Property \& Technology Law Journal, 23(1), 10-14.

The Cocktail Analysis. (2012). La $4^{\mathrm{a}}$ Oleada Observatorio de Redes Sociales en España. Recuperado de http://tcanalysis.com/blog/posts/las-marcas-empiezan-a-encontrar-limites-en-la-utilizacion-delas-redes-sociales

Twitter. (2011). Twitter about. Recuperado de http://blog.twitter.com/2011/03/numbers.html

UNIMER. (2011). Estudio de redes sociales 2011. Recuperado de http://www.unimer centroamerica. com/biblioteca_virtual/Redes_Sociales.pdf

Valenilla, Ricardo. (2011). Las marcas llegaron a las redes sociales. Debates IESA, 16(1), 54-57.

Veron, Eliseo. (1985). El análisis del "Contrato de Lectura", un nuevo método para los estudios de posicionamiento de los soportes de los media. En IREP (ed.), Les medias: Experiences, recherches actuelles, aplications. París: IREP.

Veron, Eliseo. (1988) La semiosis social: El discurso político. México: Universidad Nacional Autónoma de México, Nueva imagen.

Veron, Eliseo. (1996). De la imagen semiológica a las discursividades, en Veyrat-Masson, I. \& Dayan, D. (eds.), Espacios públicos en imágenes. Barcelona: Gedisa.

Veron, Eliseo. (1997). Esquema para el análisis de la mediatización. Diálogos, 48, 9-16.

Veron, Eliseo. (2002). Espacios mentales. Barcelona: Gedisa.

Veron, Eliseo. (2011). Papeles en el tiempo. Buenos Aires: Editorial Paidós.

Wiener, Norbert. (1950): Cibernetica y sociedad. Buenos Aires: Editorial Paidós.' 